\title{
Screening and Binding Analysis of Flavonoids with Alpha-Amylase Inhibitory Activity from Lotus Leaf
}

\author{
Liping Liao, Jing Chen, Liangliang Liu* and Aiping Xiao* \\ Institute of Bast Fiber Crops, Chinese Academy of Agricultural Sciences, 410205 Changsha, China
}

\begin{abstract}
Lotus leaf is gaining growing popularity due to its various benefits and widely usage. In this paper, ten flavonoids in lotus leaf extract were analyzed by high performance liquid chromatography (HPLC). Centrifugal ultrafiltration combined liquid chromatography was used to screen alphaamylase inhibitors from ten flavonoids mixture and the binding degrees ranged from 2.34 to $94.1 \%$. The alpha-amylase inhibition and the 1,1-diphenyl-2-picrylhydrazyl (DPPH) antioxidant activity of ten flavonoids were verified as well. Apigenin, kaempferol and isorhamnetin with relatively higher binding degrees showed higher inhibition on alpha-amylase as well. The interactions between these flavonoids and alpha-amylase were investigated by spectroscopic method. As a result, the fluorescence quenching could be considered as static quenching because the quenching rate constant $\left(\mathrm{K}_{\mathrm{q}}\right)$ values were higher than $2.0 \times 10^{10} \mathrm{~L} \mathrm{~mol}^{-1} \mathrm{~s}^{-1}$. The binding constants $\left(\log _{10} \mathrm{~K}_{\mathrm{a}}\right)$ of ten flavonoids were in the range of 5.971 to $7.417 \mathrm{~L} \mathrm{~mol}^{-1}$. The hydrogenation of $\mathrm{C}_{2}=\mathrm{C}_{3}$ double bond of apigenin and quercetin decreased the affinity for alpha-amylase (1.92- and 2.82-fold). The hydroxylation on 3 and 3' position decreased the affinity for alpha-amylase. Moreover, the glycosylation with different sugar moiety improved in varying degrees of affinity. The hydrogen bond force might be important in the binding between alpha-amylase and flavonoids.
\end{abstract}

Keywords: alpha-amylase, flavonoids, inhibitors, lotus leaf

\section{Introduction}

As a common aquatic plant, lotus is widely distributed in Asia and China. Its flower, seed, root and leaf contain plenty of dietary fibers, sugars, proteins, minerals, vitamins and many other active components and used as food additives and vegetables for a long time. ${ }^{1}$ Among them, lotus leaf is now getting popular as a food additive and traditional medicine in herbal formulations. ${ }^{2}$ It could be used for treating haematemesis, diarrhea, haematuria, sunstroke, metrorrhagia, hyperlipidaemia, fever and inflammation in traditional healthcare and modern medical treatment. The pharmacological activities such as antiviral, antiobesity, antihyperlipidemia and antimicrobial activities of lotus leaves were widely reported in recent years. ${ }^{3}$ It is reported that flavonoids are a main kind of functional components in lotus leaf possessing antioxidant activities.

The research of active compounds in natural products is more and more difficult because of the complex compositions. Related research is often hindered by tedious separation and purification procedures. ${ }^{4}$ Therefore,

*e-mail: liuliangliang@caas.cn; aipingxiao@yahoo.com efficient screening technologies for bioactive compounds are important in the research on natural products. ${ }^{5}$ In recent years, many methods and technologies were used in the screening and analysis of active compounds such as filtration, solid phase extraction and hyphenated instruments. ${ }^{6-9}$ Centrifugal ultrafiltration is a kind of membrane separation technique, which could retain high molecular weight compounds on the membrane, and low molecular weight compounds could pass through the membrane during centrifugation. ${ }^{10}$ Centrifugal ultrafiltration could be used for the screening of bioactive constituents for target biomolecules from complexes. After incubation of natural product extracts and biomolecules, the formed biomolecule-ligand complexes would be retained during ultrafiltration and be separated from the mixture. The bound ligands could be finally eluted using organic solution and be analyzed by chromatography. Compared to conventional screening and separation methods, ultrafiltration promoted the separation of ligand from unbound compounds. ${ }^{11}$ There is no need to immobilize or modify enzymes before the usage, and the use of free enzymes guaranteed its high sensitivity and selectivity. ${ }^{12}$ This method also exhibited advantages like simple 
operation steps, low sample consumption and simultaneous screening ability. ${ }^{9}$

In human body, alpha-amylase could hydrolyze starch into oligosaccharides and become one of the therapeutic targets for treating many diseases, ${ }^{13}$ especially diabetes, because they could effectively block the digestion and assimilation of carbohydrate at the early digestive absorption in body. ${ }^{14}$ In order to promote the development in related areas, many alpha-amylase inhibitors were systematically screened and reported from natural product extracts, such as Kadsura longipedunculata, pinto bean, Hibiscus sabdariffa L., Adenanthera pavonina and so on. ${ }^{15-19}$

In this study, the existence and contents of ten flavonoids were analyzed in the extract of lotus leaf. Centrifugal ultrafiltration combined with high performance liquid chromatography (HPLC) was performed for the screening and analysis of alpha-amylase inhibitors. The alpha-amylase inhibitory activities and the 1,1-diphenyl-2-picrylhydrazyl (DPPH) antioxidant activities were evaluated in order to confirm the inhibition and the antioxidant ability. The interactions between these flavonoids and alpha-amylase were further investigated by spectroscopic methods in detail. Among these flavonoids, apigenin, kaempferol and isorhamnetin showed relatively higher binding degrees and higher inhibition on alpha-amylase.

\section{Experimental}

\section{Materials}

Alpha-amylase ( $\geq 10$ units $\mathrm{mg}^{-1}$, from porcine pancreas), 3,5-dinitrosalicylic acid (DNS), 1,1-diphenyl2-picrylhydrazyl (DPPH), and soluble starch were bought from Sigma-Aldrich Chemicals (St. Louis, MO, USA). Lotus leaf was purchased from Hunan Tianjian Chinese Medicine Pieces Co., Ltd. (Changsha, China). Apigenin, hyperoside, isoquercitrin, isorhamnetin, kaempferol, luteolin, naringenin, quercetin, rutin and taxifolin were bought from Yuanye Biotechnology Co., Ltd. (Shanghai, China) with purities higher than $96.0 \%$. Acetonitrile was of HPLC grade (Tedia Inc., Phoenix, AZ, USA). Ultrapure water was purified using an ELGA water purification system (ELGA Berkefeld, Veolia, Germany). The Amicon Ultra-4 centrifugal filter was bought from Millipore (>10 kDa, Millipore, Temecula, CA, USA). The other chemicals were of analytical grade.

\section{Preparation of lotus leaf extracts}

$50.0 \mathrm{~g}$ of powdered lotus leaf was extracted in $500 \mathrm{~mL}$ of ethanol solution ( $90 \%$ in water) at $90{ }^{\circ} \mathrm{C}$ for $3 \mathrm{~h}$. After reflux extraction, $3.18 \mathrm{~g}$ of residues were obtained by removing solvent under reduced pressure. The residues were dissolved in $100 \mathrm{~mL}$ of methanol and stored at $4{ }^{\circ} \mathrm{C}$ after filtration for further usage.

\section{HPLC analysis}

HPLC analysis was conducted for the qualitative and quantitative determinations of ten flavonoids in lotus leaf extract with standard samples. The Agilent 1260 Infinity system (Agilent Technologies Inc., Santa Clara, CA, USA) equipped with a $\mathrm{C}_{18}$ reverse phase column (Waters Xbridge $^{\mathrm{TM}}, 250 \times 4.6 \mathrm{~mm}$ i.d., $5 \mu \mathrm{m}$, Milford, MA, USA) was used for the chromatographic separation. Solvent A (water containing $0.1 \% \mathrm{v} / \mathrm{v}$ acetic acid) and solvent $\mathrm{B}$ (acetonitrile containing $0.1 \% \mathrm{v} / \mathrm{v}$ acetic acid) were used as the mobile phases at $0.8 \mathrm{~mL} \mathrm{~min}^{-1}$ with gradient elution program as follow: $0-10 \mathrm{~min}, 15 \% \mathrm{~B} ; 10-45 \mathrm{~min}, 15-45 \% \mathrm{~B}$ and $45-50 \mathrm{~min}, 45 \% \mathrm{~B}$. The separation column was controlled at $25{ }^{\circ} \mathrm{C}$ and the chromatogram was recorded at $254 \mathrm{~nm}$.

\section{Screening of alpha-amylase inhibitors}

The screening was conducted using centrifugal ultrafiltration according to our previous reports with some modifications. ${ }^{20,21} 300 \mu \mathrm{L}$ of amylase $\left(1.0 \times 10^{-5} \mathrm{~mol} \mathrm{~L}^{-1}\right.$ in water) and $300 \mu \mathrm{L}$ of sample (1.0 mmol L-1 in water) were incubated at $25{ }^{\circ} \mathrm{C}$ under stirring. After $30 \mathrm{~min}$ incubation, the solution was transferred into an Amicon Ultra-4 centrifugal filter and centrifuged for $20 \mathrm{~min}$ at $10,000 \mathrm{rpm}$ and $4{ }^{\circ} \mathrm{C}$ (Beckman Coulter Allegra 64R, Brea, CA, USA). After centrifugation, $300 \mu \mathrm{L}$ of water was added to the centrifugal filter and centrifuged for $20 \mathrm{~min}$ at the same condition. This process was repeated three times to remove the unbound compounds. Finally, $300 \mu \mathrm{L}$ of $80 \%$ methanol solution was added to the centrifugal filter to elute the bound binders and then centrifuged for $20 \mathrm{~min}$. The last methanol eluent was concentrated to $100 \mu \mathrm{L}$ using nitrogen flow and stored for further analysis. Deactivated alpha-amylase after high temperature processing was used as the control solution under the same screening procedures.

The binding degree was often used as an indicator for the evaluation on the binding strength between enzyme and ligands, which could be calculated using the following equation $1: 22$

Binding degree $=\left(\mathrm{A}_{\mathrm{s}}-\mathrm{A}_{\mathrm{c}}\right) / \mathrm{A}_{0} \times 100 \%$

where $A_{0}$ is the peak area of a compound in HPLC chromatogram, $\mathrm{A}_{\mathrm{s}}$ and $\mathrm{A}_{\mathrm{c}}$ are the peak area of a compound 
after screening using active and deactivated alpha-amylase in HPLC chromatograms, respectively.

\section{Enzyme activity assay for alpha-amylase}

The activity assay for alpha-amylase was conducted using soluble starch as substrate and monitored at $540 \mathrm{~nm} .{ }^{23}$ Certain amount of sample was firstly mixed with $1 \mathrm{~mL}$ of alpha-amylase $\left(1.0 \times 10^{-5} \mathrm{~mol} \mathrm{~L}^{-1}\right.$ in water). $2 \mathrm{~mL}$ of soluble starch $\left(1.0 \mathrm{mg} \mathrm{mL}^{-1}\right)$ was then added and the mixed solution was incubated at $25^{\circ} \mathrm{C}$ for $3 \mathrm{~min}$. In order to stop the enzymatic reaction, $1 \mathrm{~mL}$ of DNS reagent was added and the solution was boiled at $100{ }^{\circ} \mathrm{C}$ for $10 \mathrm{~min}$. At last, the absorbance was measured at $540 \mathrm{~nm}$ by a UV-2600 UV-Vis Spectrophotometer (Shimadzu, Kyoto, Japan). The control experiment was conducted using water instead of enzyme solution. The alpha-amylase inhibition was calculated as follow:

Alpha-amylase inhibition $=\left(\Delta \mathrm{A}_{\mathrm{c540}}-\Delta \mathrm{A}_{\mathrm{s} 540}\right) / \Delta \mathrm{A}_{\mathrm{c540}} \times 100 \%$

where $\Delta \mathrm{A}_{\mathrm{s} 540}$ and $\Delta \mathrm{A}_{\mathrm{c} 540}$ are the absorbance increase of sample and control solution at $540 \mathrm{~nm}$, respectively. All experiments were performed thrice and the result was expressed as average value.

\section{$\mathrm{DPPH}$ free radical scavenging assay}

The DPPH free radical scavenging activity test was measured according to previous literature. ${ }^{24,25}$ Different volumes of sample $\left(5.0 \mu \mathrm{L}, 10 \mathrm{~mol} \mathrm{~L}^{-1}\right)$ and $5.0 \mu \mathrm{L}$ of DPPH solution $\left(0.1 \mathrm{mmol} \mathrm{L}^{-1}\right.$ in methanol) were diluted with methanol to $2.0 \mathrm{~mL}$. The mixed solution was vigorously shaken and incubated at $25^{\circ} \mathrm{C}$ for $30 \mathrm{~min}$ in the dark. After incubation, the absorbance was measured at $517 \mathrm{~nm}$ on a UV-2600 spectrophotometer. Methanol was substituted for flavonoid sample in the control group. The DPPH activity was calculated as follow:

DPPH activity $(\%)=\left(\mathrm{A}_{\mathrm{c517}}-\mathrm{A}_{\mathrm{s} 517}\right) / \mathrm{A}_{\mathrm{c517}} \times 100 \%$

where $\mathrm{A}_{\mathrm{s} 517}$ is the absorbance of sample at $517 \mathrm{~nm}$ and $\mathrm{A}_{\mathrm{c5} 517}$ is the absorbance of control solution at $517 \mathrm{~nm}$. The measurement was performed in triplicate and the results were reproducible within experimental error.

\section{Alpha-amylase fluorescence quenching study}

$2.0 \mathrm{~mL}$ of alpha-amylase $\left(1.0 \times 10^{-5} \mathrm{~mol} \mathrm{~L}^{-1}\right.$ in phosphate buffer $\mathrm{pH}$ 7.4) was added into a quartz cell followed by the addition of flavonoids ( $1.0 \mathrm{mmol} \mathrm{L}^{-1}$ in methanol solution).
The fluorescence spectra of alpha-amylase were recorded by a Hitachi F-7000 fluorometer (Tokyo, Japan). ${ }^{26}$ Under the excitation light at $280 \mathrm{~nm}$, the fluorescence emission spectra were recorded in the range of 300 to $450 \mathrm{~nm}$ and the fluorescence intensities at $342 \mathrm{~nm}$ were monitored for calculation. In this study, Stern-Volmer equation was used to describe the fluorescence quenching of alpha-amylase and shown as follow: ${ }^{27}$

$\mathrm{F}_{0} / \mathrm{F}=1+\mathrm{K}_{\mathrm{q}} \tau_{0}[\mathrm{Q}]=1+\mathrm{K}_{\mathrm{sv}}[\mathrm{Q}]$

where $\mathrm{F}_{0}$ is the fluorescence intensity of alpha-amylase at $342 \mathrm{~nm} ; \mathrm{F}$ is the fluorescence intensity at $342 \mathrm{~nm}$ in the presence of sample; $K_{q}$ and $K_{s v}$ are the quenching rate constant and the Stern-Volmer quenching constant, respectively; $\tau_{0}$ is the average lifetime (6.2 ns) and [Q] is the sample concentration. The binding constants were calculated by following formula:

$\log _{10}\left(\mathrm{~F}_{0}-\mathrm{F}\right) / \mathrm{F}=\log _{10} \mathrm{~K}_{\mathrm{a}}+\operatorname{nlog}_{10}[\mathrm{Q}]$

where $\mathrm{n}$ is the number of binding sites per alpha-amylase molecule and $\mathrm{K}_{\mathrm{a}}$ is the binding constant. Each test was performed in triplicate.

\section{Results and Discussion}

\section{Quantitative analysis of flavonoids}

HPLC analysis using flavonoids standards was conducted to confirm the existence of ten flavonoids in extracts of lotus leaf. As shown in Figure 1a, ten flavonoids marked as rutin (1), hyperoside (2), isoquercitrin (3), taxifolin (4), luteolin (5), quercetin (6), apigenin (7), naringenin (8), kaempferol (9) and isorhamnetin (10) were well separated and detected within $50 \mathrm{~min}$ chromatographic separation. Different concentrations of standard solutions for each compound were prepared and analyzed. The quantitative analysis was then accomplished with the standard curve of each compound. The representative chromatogram of lotus leaf extracts was shown in Figure 1b. The contents of each flavonoid were shown in Table 1 by comparing the retention time and calculating using calibration curves. Isoquercitrin and taxifolin were relatively abundant in lotus leaf extract, while apigenin, kaempferol and isorhamnetin exhibited relatively lower contents. Considering the ten flavonoids existing in lotus leaf, the further screening and analysis were conducted with the mixed flavonoids aqueous solution. 
Table 1. The structures and contents of ten flavonoids in lotus leaf extract

\begin{tabular}{|c|c|c|c|c|c|c|}
\hline \multirow{2}{*}{ No. } & \multirow{2}{*}{ Flavonoid } & \multicolumn{3}{|c|}{ Substitution } & \multirow{2}{*}{ Retention time / min } & \multirow{2}{*}{ Content / $\left(\mathrm{mg} \mathrm{g}^{-1}\right)$} \\
\hline & & $\mathrm{OH}$ & $\mathrm{OCH}_{3}$ & Others & & \\
\hline 1 & rutin & $5,7,3^{\prime}, 4^{\prime}$ & & 3-O-rutinoside & 17.3 & $1.59 \pm 0.05$ \\
\hline 2 & hyperoside & $5,7,3^{\prime}, 4^{\prime}$ & & 3-O-galactoside & 18.6 & $3.29 \pm 0.06$ \\
\hline 3 & isoquercitrin & $5,7,3^{\prime}, 4^{\prime}$ & & 3-O-glucoside & 19.3 & $29.78 \pm 0.18$ \\
\hline 4 & taxifolin & $3,5,7,3^{\prime}, 4^{\prime}$ & & flavanone & 20.4 & $10.60 \pm 0.24$ \\
\hline 5 & luteolin & $5,7,3^{\prime}, 4^{\prime}$ & & & 33.4 & $0.49 \pm 0.04$ \\
\hline 6 & quercetin & $3,5,7,3^{\prime}, 4^{\prime}$ & & & 33.8 & $2.63 \pm 0.06$ \\
\hline 7 & apigenin & $5,7,4^{\prime}$ & & & 38.8 & $0.22 \pm 0.02$ \\
\hline 8 & naringenin & $5,7,4^{\prime}$ & & flavanone & 39.5 & $1.45 \pm 0.01$ \\
\hline 9 & kaempferol & $3,5,7,4^{\prime}$ & & & 40.3 & $0.06 \pm 0.01$ \\
\hline 10 & isorhamnetin & $3,5,7,4^{\prime}$ & $3^{\prime}$ & & 40.9 & $0.39 \pm 0.04$ \\
\hline
\end{tabular}

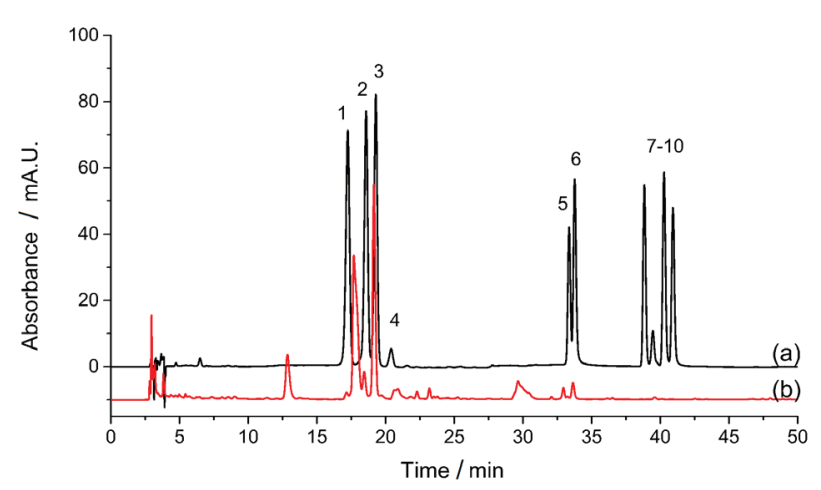

Figure 1. The chromatogram of (a) standard mixture solution of ten flavonoids (rutin (1), hyperoside (2), isoquercitrin (3), taxifolin (4), luteolin (5), quercetin (6), apigenin (7), naringenin (8), kaempferol (9) and isorhamnetin (10)); (b) lotus leaf extract.

\section{Alpha-amylase inhibitors screening from flavonoids}

The chromatograms of mixed flavonoids sample in aqueous and eluent after centrifugal ultrafiltration using active alpha-amylase were shown in Figure 2. Although the concentrations of ten flavonoids in aqueous were different from that in methanol solution because of the different solubility, ten flavonoids were apparently separated and could be observed in the chromatogram. Compared with the chromatogram of mixed flavonoids in aqueous, ten flavonoids showed various areas in the chromatogram of eluent. In the research on screening with ultrafiltration, binding degree was often used as an index to evaluate the performance of screening system and provide useful information for determining the strength of inhibitory activity. ${ }^{28,29}$ In this study, binding degrees were used to evaluate the binding performances of ten flavonoids. As shown in Table 2, the binding degree of apigenin was the highest (94.1\%). Kaempferol and isorhamnetin also showed relatively higher binding degrees (34.8 and 37.3\%, respectively).

In order to get reliable screening results, nonspecific adsorption between flavonoids and enzyme should be excluded. Therefore, the screening experiment using deactivated alpha-amylase was conducted. Figure $2 \mathrm{c}$ showed the chromatogram of eluent after centrifugal ultrafiltration with deactivated alpha-amylase. Compared with the chromatogram of eluent after centrifugal ultrafiltration using active alpha-amylase, the chromatogram of eluent using deactivated alpha-amylase showed no peak at the same retention time. Thus, the screening results using centrifugal ultrafiltration were verified and the binding of ten flavonoids could be considered as specific..$^{23,30}$

Inhibitions and DPPH activities of flavonoids in lotus leaf extracts

The alpha-amylase inhibitory activities and DPPH free radical scavenging activities of ten flavonoids were evaluated. The $\mathrm{IC}_{50}$ values of these compounds were shown in Table 2. As a result, these flavonoids exhibited various inhibition on alpha-amylase and DPPH free radical scavenging activities. Apigenin, kaempferol and 
Table 2. The binding degrees, alpha-amylase inhibition activities and DPPH free radical scavenging activities of ten flavonoids

\begin{tabular}{lcccc}
\hline No. & Flavonoid & Binding degree $/ \%$ & $\mathrm{IC}_{50} /$ alpha-amylase $\left./(\mu \mathrm{mol} \mathrm{L})^{-1}\right)$ & $\mathrm{IC}_{50} / \mathrm{DPPH} /(\mu \mathrm{mol} \mathrm{L}-1)$ \\
\hline $\mathbf{1}$ & rutin & 2.34 & 59.12 & 10.13 \\
$\mathbf{2}$ & hyperoside & 3.68 & 82.29 & 8.712 \\
$\mathbf{3}$ & isoquercitrin & 3.84 & 52.18 & 11.46 \\
$\mathbf{4}$ & taxifolin & 2.41 & 60.48 & 13.17 \\
$\mathbf{5}$ & luteolin & 8.91 & 46.81 & 15.75 \\
$\mathbf{6}$ & quercetin & 3.57 & 62.37 & 8.082 \\
$\mathbf{7}$ & apigenin & 94.1 & 25.14 & 8.246 \\
$\mathbf{8}$ & naringenin & 6.90 & 50.15 & 9.127 \\
$\mathbf{9}$ & kaempferol & 34.8 & 30.48 & 8.044 \\
$\mathbf{1 0}$ & isorhamnetin & 37.3 & 28.46 & 33.00 \\
\hline
\end{tabular}

DPPH: 1,1-diphenyl-2-picrylhydrazyl.

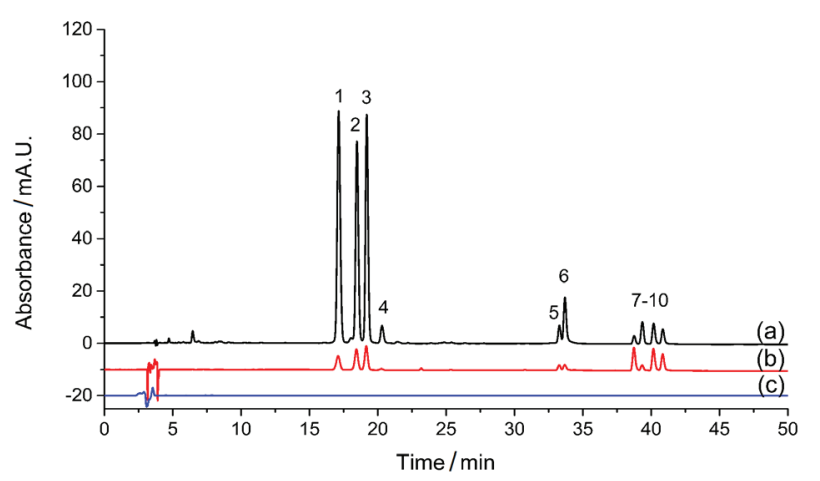

Figure 2. The chromatograms of (a) flavonoids aqueous sample; (b) eluent after centrifugal ultrafiltration using active alpha-amylase; and (c) eluent after centrifugal ultrafiltration using deactivated alpha-amylase.

isorhamnetin showed relatively higher inhibition on alphaamylase, which exhibited higher binding degrees as well. However, no obvious relationship was observed between the inhibition on alpha-amylase and the DPPH activity. These results illustrated that these flavonoids actually possess inhibition on alpha-amylase. In addition, the inhibition activities of flavonoids on alpha-amylase except taxifolin were reported according to previous research and literatures. ${ }^{31-37}$ However, the inhibition of taxifolin on alphaamylase was first screened and reported. It proved that the screening of alpha-amylase inhibitors with centrifugal ultrafiltration was effective.

\section{Quenching effects of flavonoids on alpha-amylase fluorescence}

In the fluorescence quenching study, no emission spectra of flavonoids were observed in the recorded range under excitation. The fluorescence of alpha-amylase was apparently quenched with increasing concentrations of flavonoids and the decreases varied with the kinds of samples. The Stern-Volmer plots for fluorescence quenching were further investigated for these ten flavonoids
(Figure 3). It could be seen that the Stern-Volmer plots were linear with fitting degrees ranging from 0.974 to 0.999 , which illustrated that the analyses of $\mathrm{K}_{\mathrm{sv}}$ and $\mathrm{K}_{\mathrm{q}}$ were satisfied. Based on a previous research, ${ }^{38}$ the quenching could be supposed as static quenching when $\mathrm{K}_{\mathrm{q}}$ is apparently greater than $2.0 \times 10^{10} \mathrm{~L} \mathrm{~mol}^{-1} \mathrm{~s}^{-1}$. In this study, $\mathrm{K}_{\mathrm{q}}$ values were higher than $2.0 \times 10^{10} \mathrm{~L} \mathrm{~mol}^{-1} \mathrm{~s}^{-1}$ and the fluorescence quenching could be considered as static quenching (Table 3). As shown in Table 3, the binding constant $\left(\mathrm{K}_{\mathrm{a}}\right)$ and the number of binding sites per protein molecule (n) were also calculated, and the values of $\log _{10} \mathrm{~K}_{\mathrm{a}}$ showed to be proportional to $\mathrm{n}$ (Figure 4). ${ }^{39}$ The fitting degrees of two binding constants were satisfied, ranging from 0.993 to 0.999 . The magnitudes of binding constants were in the range of $10^{5}$ to $10^{7} \mathrm{~L} \mathrm{~mol}^{-1}$.

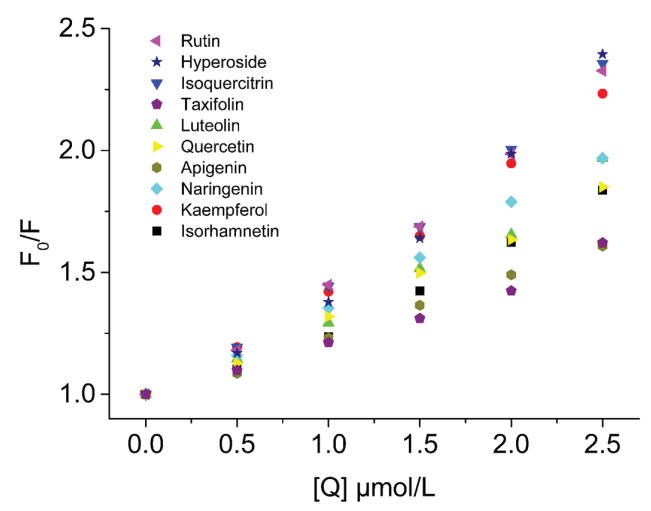

Figure 3. The Stern-Volmer plots for alpha-amylase fluorescence quenching by flavonoids.

Influence of structural alteration on the affinities for alphaamylase

\section{Hydrogenation of the $\mathrm{C}_{2}=\mathrm{C}_{3}$}

The planarity of $\mathrm{C}$ ring in flavonoids would be important for the affinity with proteins. As a consequence, the $\mathrm{C}_{2}=\mathrm{C}_{3}$ double bond plays an important role in conjugation with 
Table 3. The affinity constants of flavonoids for alpha-amylase

\begin{tabular}{|c|c|c|c|c|c|}
\hline \multirow[t]{2}{*}{ No. } & \multirow[t]{2}{*}{ Flavonoid } & \multicolumn{4}{|c|}{ Alpha-amylase affinity } \\
\hline & & $\mathrm{K}_{\mathrm{q}} \times 10^{13}$ & $\mathrm{~K}_{\mathrm{sv}} \times 10^{5}$ & $\log _{10} K_{a}$ & $\mathrm{n}$ \\
\hline 1 & rutin & 8.561 & 5.308 & 6.977 & 1.224 \\
\hline 2 & hyperoside & 8.926 & 5.534 & 7.417 & 1.303 \\
\hline 3 & isoquercitrin & 8.721 & 5.407 & 6.847 & 1.201 \\
\hline 4 & taxifolin & 3.856 & 2.391 & 5.971 & 1.109 \\
\hline 5 & luteolin & 6.076 & 3.767 & 6.474 & 1.163 \\
\hline 6 & quercetin & 5.484 & 3.400 & 6.422 & 1.158 \\
\hline 7 & apigenin & 4.043 & 2.507 & 6.634 & 1.218 \\
\hline 8 & naringenin & 6.397 & 3.966 & 6.350 & 1.134 \\
\hline 9 & kaempferol & 7.977 & 4.946 & 6.517 & 1.148 \\
\hline 10 & isorhamnetin & 5.466 & 3.389 & 7.233 & 1.306 \\
\hline
\end{tabular}

$\mathrm{K}_{\mathrm{q}}$ : quenching rate constant; $\mathrm{K}_{\mathrm{sv}}$ : Stern-Volmer quenching constant; $\mathrm{K}_{\mathrm{a}}$ : binding constant; $\mathrm{n}$ : number of binding sites per alpha-amylase molecule.

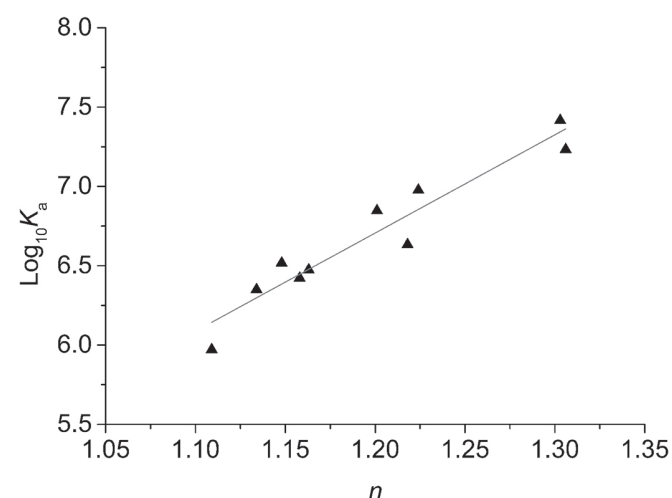

Figure 4. The relationship between calculated binding affinities $\left(\log _{10} \mathrm{~K}_{\mathrm{a}}\right)$ and number of binding sites ( $\mathrm{n}$ ) of flavonoids for alpha-amylase.

an oxo group in $\mathrm{C}$ ring. It could be seen in Table 4 that the binding affinity of flavonoids for alpha-amylase decreased after the hydrogenation of $\mathrm{C}_{2}=\mathrm{C}_{3}$ bond. Apigenin and quercetin showed higher affinities than that of naringenin and taxifolin (1.92- and 2.82-fold). The $\mathrm{B}$ ring of flavonoids could gain more twisting due to the hydrogenation of $\mathrm{C}_{2}=\mathrm{C}_{3}$ bond, which made it hard to enter the hydrophobic pockets in proteins. ${ }^{40}$

\section{Hydroxylation}

The binding constants between flavonoids and alphaamylase decreased with the hydroxylation on position 3 and 3'. It indicated that the hydroxyl group would affect the flavonoids in binding to alpha-amylase. As previous literatures reported, ${ }^{26}$ the affinities of apigenin and luteolin for alpha-amylase were 3.09 and 15.05-times higher than those of kaempferol and quercetin with the hydroxylation on position 3, and the affinity for alpha-amylase of kaempferol was 4.36-times higher than that of quercetin with the hydroxylation on position 3 '.
Table 4. Effects of structural alteration of flavonoids on the affinities for alpha-amylase

\begin{tabular}{lcc}
\hline Structural alteration & Example & Effect / times \\
\hline 2,3-Hydrogenation & apigenin $\rightarrow$ naringenin & $1.92 \downarrow$ \\
& quercetin $\rightarrow$ taxifolin & $2.82 \downarrow$ \\
\hline 3'-Hydroxylation & apigenin $\rightarrow$ luteolin & $1.44 \downarrow$ \\
& kaempferol $\rightarrow$ quercetin & $1.25 \downarrow$ \\
3-Hydroxylation & apigenin $\rightarrow$ kaempferol & $1.31 \downarrow$ \\
& luteolin $\rightarrow$ quercetin & $1.13 \downarrow$ \\
3, 3'-Hydroxylation & apigenin $\rightarrow$ quercetin & $1.63 \downarrow$ \\
& naringenin $\rightarrow$ taxifolin & $2.39 \downarrow$ \\
\hline 3-O-Glycosylation & quercetin $\rightarrow$ isoquercitrin & $2.66 \uparrow$ \\
& quercetin $\rightarrow$ hyperoside & $9.89 \uparrow$ \\
& quercetin $\rightarrow$ rutin & $3.60 \uparrow$ \\
\hline
\end{tabular}

\section{Glycosylation}

It could be seen in Table 4 that the glycosylation of flavonoids on position 3 improved the affinity for alphaamylase in different degrees depending on the difference in sugar moiety. These results could be supported by previous reports as well. ${ }^{26,40}$

\section{Conclusions}

In this study, ten flavonoids in lotus leaf extract was quantitatively analyzed. Centrifugal ultrafiltration combined with liquid chromatography was used in the screening of alpha-amylase inhibitors from ten flavonoids mixture and the binding degrees were calculated. Apigenin, kaempferol and isorhamnetin showed relatively higher inhibition on alpha-amylase and higher binding degrees. The interactions between flavonoids and alpha-amylase 
investigated by spectroscopic method showed that the fluorescence quenching was probably static quenching mode. The magnitudes of binding constants ranged from $10^{4}$ to $10^{8} \mathrm{~L} \mathrm{~mol}^{-1}$. The effects of hydrogenation of $\mathrm{C}_{2}=\mathrm{C}_{3}$ double bond, hydroxylation on 3 and 3' position and glycosylation of flavonoids on position 3 were calculated. The results showed that the hydrogen bond force might be important in the binding.

\section{Acknowledgments}

This work was supported by the Natural Science Foundation of Hunan Province (grant No. 2017JJ3348) and the risk assessment of agricultural products quality and safety project (GJFP201701003).

\section{References}

1. Guo, Y.; Chen, X.; Qi, J.; Yu, B.; J. Sep. Sci. 2016, 39, 2499.

2. Feng, C. Y.; Li, S. S.; Yin, D. D.; Zhang, H. J.; Tian, D. K.; Wu, Q.; Wang, L. J.; Su, S.; Wang, L. S.; Ind. Crop. Prod. 2016, 87, 96.

3. Li, F.; Sun, X. Y.; Li, X. W.; Yang, T.; Qi, L. W.; J. Chromatogr. B 2017, 1040, 186.

4. Lanças, F. M.; J. Braz. Chem. Soc. 2003, 14, 183.

5. Silva, M. P.; Tubino, M.; Elsholz, T. C. R.; Elsholz, O.; Khan, S.; Vila, M. M. D. C.; J. Braz. Chem. Soc. 2015, 26, 484.

6. Gaikwad, S. C.; Birla, S. S.; Ingle, A. P.; Gade, A. K.; Marcato, P. D.; Rai, M.; Duran, N.; J. Braz. Chem. Soc. 2013, 24, 1974.

7. Mantovani, S. M.; Oliveira, L. G. D.; Marsaioli, A. J.; J. Braz. Chem. Soc. 2010, 21, 1484.

8. Sargazi, A.; Aliabadi, A.; Rahdari, A.; Allahdini-Hesaroiyeh, S.; Nejati-Yazdinejad, M.; Majd, M. H.; J. Braz. Chem. Soc. 2017, 28, 950.

9. Ciesla, L.; Moaddel, R.; Nat. Prod. Rep. 2016, 33, 1131.

10. Jin, Y.; Cheng, X.; Yang, F.; Fu, L.; RSC Adv. 2015, 5, 107616.

11. Liu, S.; Yan, J.; Xing, J.; Song, F.; Liu, Z.; Liu, S.; J. Pharm. Biomed. Anal. 2012, 59, 96.

12. Li, H.; Song, F.; Xing, J.; Tsao, R.; Liu, Z.; Liu, S.; J. Am. Soc. Mass Spectrom. 2009, 20, 1496.

13. Ibrahim, S. R. M.; Mohamed, G. A.; Zayed, M. F.; Ross, S. A.; Bioorg. Chem. 2017, 70, 192.

14. Li, Y.; Gao, F.; Gao, F.; Shan, F.; Bian, J.; Zhao, C.; J. Food Sci. 2009, 74, C199.

15. Buchholz, T.; Melzig, M. F.; Phytother. Res. 2016, 30, 260.

16. Trinh, B. T. D.; Staerk, D.; Jager, A. K.; J. Ethnopharmacol. 2016, 186, 189.
17. Wickramaratne, M. N.; Punchihewa, J. C.; Wickramaratne, D. B. M.; BMC Complementary Altern. Med. 2016, 16, 5.

18. Cen, Y.; Xiao, A. P.; Chen, X. Q.; Liu, L. L.; Molecules 2016, $21,10$.

19. Ngoh, Y. Y.; Lim, T. S.; Gan, C. Y.; Enzyme Microb. Technol. 2016, 89, 76.

20. Liu, L. L.; Xiao, A. P.; Ma, L.; Li, D. F.; J. Braz. Chem. Soc. 2017, 28, 360 .

21. Liu, L. L.; Shi, S. Y.; Chen, X. Q.; Peng, M. J.; J. Chromatogr. B 2013, 932, 19.

22. Yang, Z.; Zhang, Y.; Sun, L.; Wang, Y.; Gao, X.; Cheng, Y.; Anal. Chim. Acta 2012, 719, 87.

23. Liu, L. L.; Cen, Y.; Liu, F.; Yu, J. G.; Jiang, X. Y.; Chen, X. Q.; J. Chromatogr. B 2015, 995, 64.

24. Meng, X. L.; Zheng, L. C.; Liu, J.; Gao, C. C.; Qiu, M. C.; Liu, Y. Y.; Lu, J.; Wang, D.; Chen, C. L.; RSC Adv. 2017, 7, 18347.

25. Yang, L.; Xie, X.; Yang, L.; Zhang, J.; Sun, G.; RSC Adv. 2016, $6,87616$.

26. Xiao, J.; Kai, G.; Ni, X.; Yang, F.; Chen, X.; Mol. BioSyst. 2011, 7, 1883.

27. Xiao, J.; Zhao, Y.; Wang, H.; Yuan, Y.; Yang, F.; Zhang, C.; Yamamoto, K.; J. Agric. Food Chem. 2011, 59, 10747.

28. Liu, Y.; Liu, S.; Liu, Z.; J. Chromatogr. B 2013, 923, 48.

29. Chen, M.; Liu, L.; Chen, X.; J. Sep. Sci. 2014, 37, 1546.

30. Liu, L. L.; Shi, S. Y.; Zhao, H. D.; Yu, J. G.; Jiang, X. Y.; Chen, X. Q.; J. Chromatogr. B 2014, 945, 163.

31. Choi, C. I.; Lee, S. R.; Kim, K. H.; Ind. Crop. Prod. 2015, 76, 1055.

32. Saltos, M. B. V.; Puente, B. F. N.; Faraone, I.; Milella, L.; Tommasi, N. D.; Braca, A.; Phytochem. Lett. 2015, 14, 45.

33. Zhuang, J.; Li, B.; Wang, S.; Modern Food Sci. Technol. 2011, 27,773 .

34. Wang, H.; Du, Y. J.; Song, H. C.; Food Chem. 2010, 123, 6.

35. Zeng, H.; Liu, Q.; Yu, J.; Wang, M.; Chen, M.; Wang, R.; He, X.; Gao, M.; Chen, X.; J. Sep. Sci. 2015, 38, 3897.

36. Visvanathan, R.; Jayathilake, C.; Liyanage, R.; Food Chem. 2016, $211,853$.

37. Olennikov, D. N.; Kashchenko, N. I.; Sci. World J. 2014, 2014 , 9.

38. Li, S.; Tang, L.; Bi, H.; Luminescence 2016, 31, 442.

39. Xiao, J.; Cao, H.; Wang, Y.; Yamamoto, K.; Wei, X.; Mol. Nutr. Food Res. 2010, 54, S253.

40. Xiao, J.; Ni, X.; Kai, G.; Chen, X.; Crit. Rev. Food Sci. Nutr. 2013, 53, 497.

Submitted: July 31, 2017 Published online: September 25, 2017 